\title{
Recent Developments in Radiation Oncology: An Overview of Individualised Treatment Strategies in Breast Cancer
}

\author{
Montserrat Pazos ${ }^{a}$ Stephan Schönecker ${ }^{a}$ Daniel Reitz ${ }^{a} \quad$ Paul Rogowski $^{a} \quad$ Maximilian Niyazi ${ }^{a}$ \\ Filippo Alongi ${ }^{b, c}$ Christiane Matuschek ${ }^{d} \quad$ Michael Braun $^{e} \quad$ Nadia Harbeck $^{f}$ \\ Claus Belka ${ }^{a}$ Stefanie Corradini ${ }^{\mathrm{a}}$ \\ a Department of Radiation Oncology, University Hospital, LMU Munich, Munich, Germany; \\ ${ }^{b}$ Department of Radiation Oncology, Sacro Cuore Don Calbria Negrar, Verona, Italy; \\ ' University of Brescia, Brescia, Italy; \\ ${ }^{\mathrm{d}}$ Department of Radiation Oncology, Heinrich Heine University, Düsseldorf, Germany; \\ e Department of Gynecology and Obstetrics, Red Cross Hospital, Munich, Germany; \\ ${ }^{f}$ Breast Center, Department of Gynecology and Obstetrics, University Hospital, LMU Munich, Munich, Germany
}

\section{Keywords}

Breast cancer · Individualised therapy .

Hypofractionation · Risk-adapted

\section{Summary}

Radiation therapy (RT) for breast cancer has dramatically changed over the past years, leading to individualized risk-adapted treatment strategies. Historically, the choice of $\mathrm{RT}$ regimen was limited to conventional fractionation protocols using standard tangential fields. Nowadays, technological and technical improvements in modern RT have added a variety of other RT modalities, different fractionation schedules, and individualised treatment volumes to the portfolio of breast RT. This review aims to give a short overview on the main topics which have recently found their way into clinical practice: hypofractionated treatment protocols, accelerated partial breast irradiation (APBI) for low-risk patients, deep inspiration breath hold (DIBH) for maximal heart protection, extent of regional nodal irradiation for high-risk patients, and the implementation of new radiation techniques such as intensity modulated RT (IMRT) and volumetric modulated RT (VMAT).

(C) 2018 S. Karger GmbH, Freiburg

M. Pazos and S. Schönecker contributed equally to this work.

\section{Introduction}

Radiation therapy (RT) plays a key role in most curative approaches of breast cancer (BC) management $[1,2]$. RT following breast-conserving surgery (BCS) allows to halve the risk of ipsilateral breast recurrences and reduce BC-specific mortality. Moreover, RT represents an essential part of treatment in the locallyadvanced setting following mastectomy [3]. Over several decades, the choice of RT regimen was limited to a conventional fractionation protocol over 5 weeks ( \pm boost to the surgical bed) using opposing tangential coplanar fields covering the whole breast or the chest wall. Nowadays, technological and technical improvements in modern RT have added a variety of other RT modalities (intensity modulated radiotherapy (IMRT)/volumetric modulated radiotherapy (VMAT), deep-inspiration breath-hold (DIBH)), different fractionation schedules (hypofractionation, simultaneous integrated boost (SIB)), and individualised treatments (e.g., accelerated partial breast irradiation (APBI)) to the portfolio of breast $\mathrm{RT}$. The aim of this review is to give an overview of recent developments in RT for BC and to address clinical and technological aspects of their implementation in daily practice.

\section{Hypofractionated Radiotherapy}

START A, START B, and the Canadian trial delivered clear data in favour of hypofractionation in the treatment of early-stage BC [4-7]. Before these studies, the standard of adjuvant care was represented by conventional fractionated radiotherapy (CFRT) using

\section{KARGER}

() 2018 S. Karger GmbH, Freiburg 
Table 1. Important recent trials regarding hypofractionation in breast cancer

\begin{tabular}{|c|c|c|c|c|c|c|c|c|c|}
\hline & \multicolumn{3}{|c|}{ START-A [4] } & \multicolumn{3}{|c|}{ START-B [4] } & \multicolumn{3}{|c|}{ Canadian Trial [7] } \\
\hline & CFRT & HFRT & $\mathrm{p}$ & CFRT & HFRT & $\mathrm{p}$ & CFRT & HFRT & $\mathrm{p}$ \\
\hline Patients, $\mathrm{n}$ & 749 & $737 / 750$ & & 1,105 & 1,110 & & 612 & 622 & \\
\hline Median follow-up, years & \multicolumn{2}{|c|}{9.3} & & \multicolumn{2}{|c|}{9.9} & & \multicolumn{2}{|c|}{12.0} & \\
\hline Daily RT dose, Gy & 2 & $3.0 / 3.2$ & & 2 & 2.67 & & 2 & 2.66 & \\
\hline Total RT dose, Gy & 50 & $39.0 / 41.6$ & & 50 & 40.05 & & 50 & 42.5 & \\
\hline Mean treatment time, days & 35 & $35 / 35$ & & 35 & 21 & & 35 & 22 & \\
\hline 10 -year locoregional relapse, $\%$ & 7.4 & $8.8 / 6.3$ & $0.41 / 0.65$ & 5.5 & 4.3 & 0.21 & 6.7 & 6.2 & $0.001^{\mathrm{a}}$ \\
\hline 10 -year overall survival, $\%$ & 80.2 & $79.7 / 81.6$ & $0.69 / 0.74$ & 80.8 & 84.1 & 0.042 & 84.4 & 84.6 & 0.79 \\
\hline
\end{tabular}

${ }^{a}$ For non-inferiority.

$\mathrm{CFRT}=$ Conventional fractionated radiation therapy; HFRT $=$ hypofractionated radiation therapy; $\mathrm{RT}=$ radiotherapy.

1.8/2 Gy per fraction up to a dose of 50.4/50 Gy. The UK START trial A randomized 2,236 women with early BC after BCS between CFRT (50 Gy in 2.0-Gy fractions) and hypofractionated radiotherapy (HFRT) (41.6 Gy in 3.2-Gy or 39 Gy in 3-Gy fractions over 5 weeks), and the UK START trial B to a hypofractionated and accelerated scheme (40 Gy in 2.67-Gy fractions over 3 weeks). In 2013, the updates of the UK START trials were published [4]: The median follow-up was 9.3 years (START A) and 9.9 years (START B). Regarding the primary endpoint of locoregional tumour relapse, there were no significant differences between the groups. Interestingly, the hypofractionation groups showed a trend towards a lower risk for ipsilateral recurrence (hazard ratio (HR) 0.91, p = 0.65 and HR 0.77, p = 0.21). Regarding late normal tissue effects, the $39-$ Gy group as well as the $40-$ Gy group showed significantly less breast induration/shrinkage, telangiectasia, and breast oedema as compared to CFRT. Moreover, regarding cardiac events, there were no major differences in the groups of patients with left-sided primary tumours. A further reduction in the overall treatment time is currently being analysed in the UK FAST [5] and FAST-FORWARD [6] trials. The third randomized trial was conducted in Canada [7]: 1,234 early BC patients were randomly assigned to either CFRT ( 5 weeks) or HFRT with 42.5 Gy in 16 fractions of 2.66 Gy. At the 10-year follow-up, the HFRT group showed an absolute difference of $-0.5 \%$ local recurrence rate compared to the CFRT group (6.2 vs. $6.7 \% ; \mathrm{p}<0.001$ for non-inferiority); overall survival (OS) was also not significantly different $(\mathrm{p}=0.79)$, and the cosmetic outcome was similar between the 2 treatment groups. A detailed overview is given in table 1.

In a systematic review based on these data, Zhou et al. [8] suggested that HFRT with 2.5-3.0 Gy per fraction should be the better choice for the treatment of early BC patients. HFRT was associated with decreased grade $2 / 3$ acute skin reactions and significantly decreased moderate/marked photographic changes in breast appearance compared to CFRT (relative risk (RR) 0.80 , 95\% confidence interval (CI) $0.70-0.91 ; \mathrm{p}=0.001$ ). Regarding locoregional recurrence, distant metastasis, OS, disease-free survival (DFS), symptomatic radiation pneumonitis, ischemic heart disease, and symptomatic rib fracture, there were no significant differences between HFRT and CFRT. These findings were also confirmed in a recently published meta-analysis of the randomized trials that included more than 8,000 patients [9].

Following mastectomy, or in the management of women requiring regional nodal irradiation (RNI), there is less evidence for HRFT. In the START A/B trials, 491 patients had a mastectomy and received hypofractionated postmastectomy radiotherapy (HFPMRT). The first prospective phase II study on HF-PMRT was recently published in May 2017 [10] showing promising results. The same group is carrying out the Alliance A221505 study, a phase III randomized trial of HF-PMRT following breast reconstruction. Similarly, patients requiring RNI were underrepresented in randomized trials. Subgroup analyses from these randomized trials and smaller prospective studies support the feasibility of hypofractionated RNI $[11,12]$. On the other hand, randomized data with adequate long-term follow-up to evaluate possible toxicities in organs at risk (e.g., brachial plexus) are pending and preclude drawing definitive conclusions.

In Germany, the updated S3 guideline for BC has introduced hypofractionation as the standard treatment option after BCS if lymph node irradiation is not recommended. To date, if RNI is necessary, CFRT still represents the treatment of choice.

\section{Boost}

Two large randomized trials $[13,14]$ documented the positive effect of localized dose escalation on local control when using a boost to the tumour bed, while no effect on long-term OS was found. Romestaing et al. [14] randomized 1,024 patients with early BC between 1986 and 1992 to receive either a boost of 10 Gy (4× 2.5 Gy with electrons) after whole breast irradiation (WBI) with $50 \mathrm{~Gy}$ or no further dose escalation. At 5 years, there was a significant reduction in local relapses $(\mathrm{p}<0.044)$ with no serious deterioration in the cosmetic results. Similarly, a randomized trial of 2,657 patients studied the impact of a boost of 16 Gy $(8 \times 2$ Gy with either electrons, photons, or brachytherapy). After a 20 -year follow-up, there was a significant reduction in the cumulative incidence of ipsilateral breast recurrences in the boost group with a moderate increase in severe fibrosis (1.8 vs. 5.2\%) [14]. Impaired 
cosmetic results were described for boost volumes $>200 \mathrm{cc}$ [15] Nevertheless, according to a recent Cochrane review that included 8,325 patients from several randomized controlled trials, the objective percentage of breast retraction assessment appears similar between patients with and without boost [16]. Furthermore, the application of the boost reduced the number of salvage mastectomies by more than a third compared to standard treatment with WBI [15]. The effect of the additional boost seemed to be independent of tumour characteristics such as tumour grade or additional adjuvant systemic treatments, as reported 2001 by Bartelink et al. [17]. From today's point of view, this conclusion has to be interpreted with caution, as the EORTC (European Organisation for Research and Treatment of Cancer) study was conducted before the era of neoadjuvant systemic therapy and the introduction of targeted therapies (e.g., HER2 blockade). With regard to histology, the presence of ductal carcinoma in situ adjacent to invasive tumour was associated with higher local relapse rates in women $<50$ years of age, according to the most recent publication of the EORTC trial [18]. For this group of patients, the additional boost significantly reduced local recurrences from 31 to $15 \%$ at 20 -year follow-up [18].

The relative benefit of the boost dose for local control was independent of age; however, with increasing age, the absolute gain in local control decreased. The largest benefit was found for young patients $<50$ years of age [14]. As the absolute benefit decreases with increasing age, the indication for a boost should be established individually taking tumour biology and risk of local recurrence into account. There are alternative technical methods to escalate the dose in the tumour bed apart from a sequential boost using external beam radiotherapy (EBRT) with photons or electrons: intraoperative radiation therapy (IORT/intraoperative electron radiation therapy (IOERT)), brachytherapy, or SIB.

The IORT/IOERT boost applies a high single fraction (20 Gy $50 \mathrm{kV}$ X-rays for IORT, 10-12 Gy electrons for IOERT) during surgery. The advantage of both techniques is the direct visualisation of the tumour bed, which lowers the risk of a geographic miss. As a consequence of the direct tissue exposure without postoperative distension by hematoma/seroma, IORT/IOERT theoretically also allows for a smaller treatment volume. Two known disadvantages are that an intraoperative boost prolongs the surgical procedure and that conventional fractionated WBI is usually still delivered after surgical wound healing. The combination with hypofractionated EBRT is currently under evaluation in 2 multicentric prospective trials: as $\mathrm{kV}$-IORT in the TARGIT-B(oost) study and as IOERT in the HIOB trial (3 weeks hypofractionated WBI preceded by IORT electron boost) [19].

Over the last few years, several groups have reported outcomes after a boost applied simultaneously during conventional fractionated WBI. This SIB can be planned either by using 3-dimensional (3D) conventional techniques or modern modulated IMRT/VMAT techniques. The rationale is a localized dose escalation in the area most at risk for recurrence without prolonging the overall treatment duration [20]. From the published series, daily tumour bed doses between 2.1 Gy for low-risk and 2.25 Gy for high-risk set- tings seem to be within the therapeutic range [20]. However, prospective data on long-term toxicity are still not available.

Although the combination of hypofractionated WBI and simultaneous boost has been evaluated in some trials [21], this approach is discouraged outside clinical trials due to the absence of robust follow-up data. The RTOG-1005 phase III trial has finished patient accrual. This trial compared a sequential treatment $(15 \times 2.67 \mathrm{~Gy}$ WBI followed by $5 \times 2$-Gy boost) with a concomitant boost schedule $(15 \times 2.67$ Gy WBI/15 × 3.2-Gy SIB $)$ [22]. In Germany, Austria, and Switzerland, the current HYPOSIB trial tests a hypofractionated RT with SIB $(16 \times 2.5 \mathrm{~Gy} \mathrm{WBI} / 16 \times 3-\mathrm{Gy}$ SIB $)$ following the dose prescription of a phase II trial with 151 patients [23]. The end of recruitment is expected for 2019 .

\section{Accelerated Partial Breast Irradiation}

Over the recent years, there has been an increasing interest in developing treatment strategies using APBI, especially for patients with low-risk tumours. Overall, recurrence rates in the APBI studies are very low. Most trials have demonstrated increased rates of local recurrences, but no difference in OS [24]. The rationale for this approach is based on the knowledge that most local in-breast recurrences are located very close to the initial tumour site (within a 1 - to $2-\mathrm{cm}$ radius), and the rate of relapses outside the tumour bed area seems to be the same as the recurrence rate in the contralateral breast [25]. APBI has been administered using different techniques (brachytherapy, IORT/IOERT, EBRT), applying different doses and fractionation schedules, and using various target volume definitions [24]. A summary of results and patient characteristics of the recent randomized trials of APBI versus WBI has been recently published in a review by Krug et al. [26]

Taken together, multicatheter brachytherapy with its 10 -year follow-up is the technique with the longest clinical experience for APBI. 5-year side effects and cosmetic results have recently been published [27]. The TARGIT-A trial and the ELIOT trial tested IORT with $50 \mathrm{kV}$ X-ray or electrons. Although non-inferiority was established in both trials, there are some methodologic issues with the design of these studies. Furthermore, long-term results of 3 phase III trials for APBI with EBRT are awaited within the next years. The results of one of these trials (IMPORT Low [28]) demonstrated non-inferiority for the experimental arm; however, in the RAPID trial (preliminary data), which used a higher daily dose, the cosmetic results seem to be inferior in the experimental arm. The authors themselves advice against the use of this technique outside clinical trials. It can be concluded that long-term follow-up is needed to assess the impact of APBI [26] and that for this treatment strategy, selection of adequate low-risk patients is crucial.

\section{Postmastectomy Radiotherapy}

A meta-analysis by the Early Breast Cancer Trialists' Collaborative Group (EBCTCG) containing data of 8,135 women from 22 
randomized trials showed robust evidence for PMRT for high-risk patients with more than 4 tumour-infiltrated lymph nodes, as well as for intermediate-risk patients with 1-3 positive lymph nodes [3]. It showed a significant reduction in both overall recurrence and BC mortality in patients with $1-3$ positive lymph nodes (overall recurrence: RR 0.68, 95\% CI 0.57-0.82; $\mathrm{p}=0.00006$ and BC mortality: RR $0.80,95 \%$ CI $0.67-0.95 ; \mathrm{p}=0.01$ ) or with $\geq 4$ positive lymph nodes (overall recurrence: RR 0.79, 95\% CI 0.69-0.90; $\mathrm{p}=0.0003$ and BC mortality: RR 0.87, 95\% CI 0.77-0.99; $\mathrm{p}=0.04$ ).

The updated German S3 guideline recommends PMRT in the setting of advanced tumour stage T3 $(>5 \mathrm{~cm})$ and T4 tumours independent of the lymph node status. One exception are pT3 pN0 tumours with clear resection margins and no additional risk factors like lymphangiosis (L1), high tumour grade (G3), premenopausal status, young age $<50$ years, or negative hormone receptor status [29]. Patients with positive resection margins (R1 or R2) should always receive PMRT. With regard to lymph node involvement, PMRT is recommended for all high-risk patients with 4 or more positive lymph nodes [30]; for patients with 1-3 positive lymph nodes, the individual risk for local recurrence should be taken into account and discussed interdisciplinarily. High-risk features include: high tumour grade (G3), triple-negative receptor status, multifocal tumour, lobular histology, lymphangiosis, high Ki-67 > 30\%, young age $<45$ years, medial tumour location, or tumour size $>2 \mathrm{~cm}$.

After neoadjuvant chemotherapy (NACT), the decision for PMRT is still under debate. The updated S3 guidelines state that the indication for adjuvant RT should be based on the pre-therapeutic staging ( $\mathrm{cN}+, \mathrm{cT} 3 / \mathrm{cT} 4 \mathrm{a}-\mathrm{d})$; however, in the case of pathologic complete remission, interdisciplinary discussion based on individual risk factors is advised. Recently published studies from Korea and France have contributed to the hypothesis that the recurrence risk may be low enough to omit RT in selected patients with stage II-III $\mathrm{BC}$ after a favourable response to NACT and mastectomy [31, 32]. Other publications have found a positive effect of PMRT for all cN+ patients following NACT, such as Rusthoven et al. [33] whose study included women with cT1-3 cN1 M0 BC treated with mastectomy after NACT (3,040 ypN0 and 7,243 ypN+ cases). PMRT improved $\mathrm{OS}$ in univariate and multivariate analyses for $\mathrm{ypN}+$ as well as for ypN0 cases. Looking separately at the different pathologic nodal subgroups (ypN0, ypN1, and ypN2), there was a significant OS benefit for PMRT for all of them. The ongoing prospective randomized trials NSABP B51/RTOG 1304 and ALLIANCE A011202 will clarify some of the issues regarding local treatments after NACT. Until then, clinicians should discuss every case individually in a multidisciplinary setting, taking into account the various aspects of efficacy and side effects to avoid over-irradiation [34].

Another important aspect when indicating PMRT is the timing of RT according to the type of breast reconstruction [35, 36]. Historically, breast reconstruction was performed after all oncological treatments had been completed (delayed reconstruction). Over the past decades, immediate breast reconstruction (IBR) after skinsparing mastectomy (SSM) has gained wide acceptance. IBR has a number of advantages over delayed reconstruction but can be negatively influenced by PMRT regarding cosmetic outcome. In order to avoid complications of RT in conjunction with IBR, several solutions are described in literature: a delayed immediate reconstruction where a temporary implant is placed for the time of RT and replaced with an implant or autologous tissue following RT, or a neoadjuvant/premastectomy RT strategy where RT is delivered before SSM and IBR [36-38].

\section{Regional Nodal Irradiation}

At present, the role of axillary lymph node dissection (ALND) is decreasing in the treatment of early $\mathrm{BC}$, and its potential advantages/disadvantages over sentinel lymph node biopsy (SLNB) have been widely debated over the past years [39]. In the AMAROS trial [40], both ALND and axillary RT, after positive SLNB, provided excellent and comparable axillary control rates for patients with early-stage BC and no palpable lymphadenopathy. Moreover, axillary RT resulted in significantly reduced morbidity due to the fact that ALND included the removal of level 3 nodes, which caused a high percentage of lymphedema.

The publication of the American College of Surgeons Oncology Group (ACOSOG) Z0011 trial [41] confirmed the very low overall axillary recurrence rates $(0.9 \%)$ of SLNB-positive patients who did not undergo ALND. The results suggested that in patients with early-stage BC and low axillary tumour burden, administration of adjuvant WBI and systemic therapy is a sufficient treatment to obtain satisfactory locoregional control. Although the ACOSOG Z0011 protocol specified standard WBI by tangential fields without any lymph node irradiation, detailed information on the extent of RT volumes was not published initially. This raised the question whether the low regional recurrence rates observed in Z0011 were actually caused by the use of so-called 'high tangents'. By enlarging the standard tangential fields on the cranial border, axillary coverage can be improved significantly [41]. In 2014, Jagsi et al. [42] published available details on RT volumes (1/3 of all patients), showing that $50 \%$ of Z0011 patients had received 'high tangents' and $15 \%$ an additional RNI to the supraclavicular region, with no differences between the groups.

The ongoing German INSEMA study (NCT02466737) is currently investigating the feasibility and safety of omission of the SNLB. Patients with early-stage BC and a clinically negative axilla are randomized to receive SLNB or no axillary intervention at all. If the SLNB is positive, a second randomization (ALND vs. no ALND) will follow. Besides local outcome, a secondary objective of the study is to clearly assess the RT dose that reaches the different axillary levels during standard WBI.

Positive support for RNI in all lymph node-positive patients was recently provided by the National Cancer Institute of Canada MA.20 trial [43] that demonstrated an improved 10-year locoregional recurrence-free survival (95.2 vs. $92.2 \% ; \mathrm{p}=0.009$ ) and DFS ( 82 vs. $77 \% ; \mathrm{p}=0.01$ ) for RNI in node-positive (85\%) or 'high-risk' node-negative (10\%) patients after BCS. Patients were randomized to WBI \pm RNI (including axillary, supraclavicular, and internal mammary nodes (IM-LN)). The two other randomized studies, 
Table 2. Important recent trials regarding regional nodal irradiation in breast cancer

\begin{tabular}{|c|c|c|c|c|c|c|c|c|c|c|c|c|}
\hline & \multicolumn{3}{|c|}{ EORTC 22922/10925 } & \multicolumn{3}{|l|}{ MA.20 } & \multicolumn{3}{|c|}{ French Trial [45] } & \multicolumn{3}{|l|}{ DBCG-IMN [47] } \\
\hline & $\begin{array}{l}\text { WBI + } \\
\text { boost } \\
\text { TWI }\end{array}$ & $\begin{array}{l}\text { WBI + boost + } \\
\text { SCV/IMN } \\
\text { TWI + } \\
\text { SCV/IMN }\end{array}$ & $\mathrm{p}$ & $\begin{array}{l}\text { WBI + } \\
\text { boost }\end{array}$ & $\begin{array}{l}\text { WBI + boost + } \\
\text { SCV/IMN }\end{array}$ & $\mathrm{p}$ & $\begin{array}{l}\text { TWI + } \\
\text { SCV }\end{array}$ & $\begin{array}{l}\text { TWI + } \\
\text { SCV/IMN }\end{array}$ & $\mathrm{p}$ & $\begin{array}{l}\text { WBI + boost + } \\
\text { SCV } \\
\text { (left-sided } \\
\text { tumour) }\end{array}$ & $\begin{array}{l}\text { WBI + boost + } \\
\text { SCV/IMN } \\
\text { (right-sided } \\
\text { tumour) }\end{array}$ & $\mathrm{p}$ \\
\hline Patients, $\mathrm{n}$ & \multicolumn{3}{|l|}{4,004} & \multicolumn{3}{|l|}{1,832} & \multicolumn{3}{|l|}{1,334} & \multicolumn{3}{|l|}{3,089} \\
\hline $\begin{array}{l}\text { Median follow-up, } \\
\text { years }\end{array}$ & \multicolumn{3}{|l|}{10.9} & \multicolumn{3}{|l|}{9.5} & \multicolumn{3}{|l|}{8.6} & \multicolumn{3}{|l|}{8.9} \\
\hline Inclusion criteria & \multicolumn{3}{|c|}{$\begin{array}{l}\mathrm{N}+\text { or } \mathrm{N} 0 \\
\text { (medial or central } \\
\text { tumour location) }\end{array}$} & \multicolumn{3}{|c|}{$\begin{array}{l}\mathrm{N}+\text { or } \mathrm{N} 0 \text { high risk } \\
\text { (T3 or } \mathrm{T} 2 \text { with }<10 \\
\text { resected lymph nodes } \\
\text { and additional risk } \\
\text { factors present) }\end{array}$} & \multicolumn{3}{|c|}{$\begin{array}{l}\mathrm{N}+\text { or } \mathrm{N} 0 \\
\text { (medial or central } \\
\text { tumour location) }\end{array}$} & \multicolumn{3}{|l|}{$\mathrm{N}+$} \\
\hline Overall survival, \% & 80.7 & 82.3 & 0.06 & 81.8 & 82.8 & 0.38 & 59.3 & 62.6 & 0.8 & 72.2 & 75.9 & 0.005 \\
\hline
\end{tabular}

EORTC 22922/10925 [44] and the French study by Hennequin et al. [45], had similar but not identical inclusion criteria. Most of the patients had positive nodes and/or medially located tumours. In the French study, all patients received RNI to the supraclavicular nodes and were randomized for RT to the IM-LN. Overall, there was no significant survival benefit for IM-LN RT (10-year OS 62 vs. 59\%) [45]. The EORTC trial randomized patients who had undergone either BCS (76\%) or mastectomy (24\%) to WBI/chest wall \pm RNI (medial supraclavicular nodes and IM-LN). RNI improved local, distant, and overall outcome at 10 years: OS: 82 vs. $80 \%, \mathrm{p}=$ 0.049; DFS: 72.1 vs. $69 \%, \mathrm{p}=0.044$ [44]. Most patients in the trials received additional systemic chemotherapy or endocrine treatment according to standard recommendations at the time of patient recruitment (French: 1991-1997; EORTC: 1996-2004; MA.20: 20002007). With the exception of the MA.20 trial, however, the study protocols are outdated compared to current standards, as old radiation techniques and chemotherapy regimens were used (table 2).

A meta-analysis of the 3 trials by Budach et al. [46] in 2015 detected an even more distinct benefit of RNI on OS. RNI to the supraclavicular lymph nodes and IM-LN (MA.20 and EORTC) resulted in a significant improvement in OS (HR 0.88, 95\% CI $0.78-0.99)$.

The gain in DFS and distant metastasis-free survival resulted in an absolute OS benefit at 10 years of $1 \%$ in the MA.20 trial, 1.6\% in the EORTC trial, and 3.3\% in the French trial (not significant in single trials). The fear of an increase in cardiovascular toxicity due to RNI to the IM-LN has not been confirmed after 10-years of follow-up. Also, lung toxicity and lymphedema risk were slightly increased but manageable. Further follow-up is currently awaited.

Recently, a prospective population-based cohort study [47] conducted in Denmark (DBCG-IMN) added some valuable information to the discussion about RNI to IM-LN. In this study, all nodepositive patients received RT to the supra-/infraclavicular region and only patients with right-sided tumours also received IM-LN irradiation. The addition of IM-LN irradiation increased OS in patients with early-stage node-positive $\mathrm{BC}$ at 8 years from 72.2 to $75.9 \%$ (HR 0.82, $\mathrm{p}<0.005)$.

\section{Role of Modern Techniques: IMRT, VMAT, and DIBH}

As already mentioned, RT techniques have substantially improved over the last 20 years. Historically, RT regimens were known to increase long-term overall mortality from secondary lung cancer and heart disease through radiation exposure [48]. Advanced modulated RT techniques such as VMAT or IMRT result in better dose homogeneity within the target volume and allow for a significant dose reduction for organs at risk (e.g., heart) [49]. Moreover, IMRT/VMAT has dosimetric advantages as compared to $3 \mathrm{D}$ conformal radiotherapy (3D-CRT) in the case of irradiation of complex volumes (e.g., IM-LN RNI) or application of a simultaneous boost. In clinical practice, modulated RT techniques are still not considered standard of care, despite some studies having shown a reduction in early and long-term side effects as compared to 3D-CRT. Nevertheless, breast IMRT/VMAT may be useful for selected patients [50].

Recent data show that even low radiation doses to the heart can play a relevant role in the development of late cardiac toxicity after $\mathrm{BC}$ treatment $[51,52]$. However, neither the exact pathomechanisms nor the exact dose-response relationships or the critical regions within the heart are well defined. To date, no reasonable 'safe' threshold dose for late cardiac morbidity or mortality is established [51]. Therefore, and in light of the increasing use of systemic therapies with potentially cardiotoxic agents, it is particularly important to reduce the cardiac dose to the lowest possible level. Respiratory gating by using a breath-hold procedure is an emerging tool in RT and is considered a safe, feasible, and easily reproducible solution to mitigate intrafractional breathing motion dur- 
ing each treatment fraction. During DIBH, the patient inhales to a specified threshold and successively holds her breath at a specific level of inspiration during the delivery of RT. The use of this technique, for example for BC irradiation, has been associated with a lower dose exposure of the heart without compromising target volume coverage [53-55]. Currently, several voluntary or computercontrolled techniques from different suppliers are available.

\section{Elderly Patients at Low Risk}

As older patients tend to be excluded from clinical trials, randomized evidence for elderly patients receiving locoregional treatment is limited. The results of the CALGB 9343 trial [56] showed $98 \%$ local control in the group of patients older than 70 years treated with RT and tamoxifen versus $90 \%$ in those receiving tamoxifen alone, with no significant difference in OS for early-stage oestrogen receptor-positive BC. Given the small absolute benefit of RT, coupled with its potential for morbidity, new clinical guidelines have included omission of RT as an option for elderly women. Yet, according to the PRIME-II trial [57] which also randomized patients older than 65 years to RT and endocrine treatment versus endocrine treatment alone, no overall difference in quality of life (Euro QoL measurements) was seen within the first 15 months of follow-up and 5 years afterwards. On this basis, the fear of side effects should not be the main criterion for omitting postoperative RT in elderly patients.

Evaluation of RT strategies in elderly patients should be handled with caution, taking into account tumour biology, comorbidities, performance status, and patient preferences [58]. For example, elderly patients with oestrogen receptor-negative $\mathrm{BC}$ were found to have a significant reduction in 5-year BC-specific death in an observational study by Eaton et al. [59] following adjuvant RT (10.8 and $24.1 \%, \mathrm{p}<0.001)$. In this regard, modern adjuvant RT strategies, such as APBI or IORT, may become an attractive treatment alternative for selected elderly patients. Furthermore, HFRT regimens may be more convenient for elderly patients and are supported by level 1 evidence [60].

\section{Conclusion}

$\mathrm{RT}$ is in the midst of a radical change based on recent technological innovations and clinical information provided continuously by new randomized evidence from clinical trials. Nowadays, radiation oncology strategies for BC patients are more individualised and risk-adapted taking into account tumour and patient characteristics. While APBI has become a well-recognized treatment option for selected low-risk patients, HFRT is a new standard of care for WBI. Other modern radiation techniques, including IMRT/ VMAT and DIBH, have been introduced with the aim of reducing high doses to healthy tissues and organs at risk (e.g., heart) during RT or allowing for a simultaneous application of a boost to the tumour bed. Furthermore, regarding the irradiation of the lymphatic pathways, risk-adapted strategies have also been introduced into clinical practice.

\section{Disclosure Statement}

The authors declare that they have no competing interests.

\section{References}

1 Corradini S, Niyazi M, Niemoeller OM, et al: Adjuvant radiotherapy after breast conserving surgery - a comparative effectiveness research study. Radiother Oncol 2015;114:28-34.

2 Veronesi U, Zucali R, Luini A: Local-control and survival in early breast-cancer - the Milan trial. Int J Radiat Oncol Biol Phys 1986;12:717-720.

$\checkmark 3$ EBCTCG (Early Breast Cancer Trialists' Collaborative Group); McGale P, Taylor C, et al: Effect of radiotherapy after mastectomy and axillary surgery on 10-year recurrence and 20-year breast cancer mortality: metaanalysis of individual patient data for 8135 women in 22 randomised trials. Lancet 2014;383:2127-2135.

$\checkmark$ Haviland JS, Owen JR, Dewar JA, et al: The UK Standardisation of Breast Radiotherapy (START) trials of radiotherapy hypofractionation for treatment of early breast cancer: 10-year follow-up results of two randomised controlled trials. Lancet Oncol 2013;14:10861094.

$\checkmark 5$ FAST Trialists group, Yarnold JR, Agrawal RK, et al: First results of the randomised UK FAST Trial of radiotherapy hypofractionation for treatment of early breast cancer (CRUKE/04/015). Radiother Oncol 2011; 100:93-100.
Brunt AM, Wheatley D, Yarnold J, et al: Acute skin toxicity associated with a 1-week schedule of whole breast radiotherapy compared with a standard 3-week regimen delivered in the UK FAST-Forward Trial. Radiother Oncol 2016;120:114-118.

7 Whelan TJ, Pignol JP, Levine MN, et al: Long-term results of hypofractionated radiation therapy for breast cancer. N Engl J Med 2010;362:513-520.

8 Zhou ZR, Mei X, Chen XX, et al: Systematic review and meta-analysis comparing hypofractionated with conventional fraction radiotherapy in treatment of early breast cancer. Surg Oncol 2015;24:200-211.

$\checkmark$ Valle LF, Agarwal S, Bickel KE, Herchek HA, Nalepinski DC, Kapadia NS: Hypofractionated whole breast radiotherapy in breast conservation for early-stage breast cancer: a systematic review and meta-analysis of randomized trials. Breast Cancer Res Treat 2017;162: 409-417.

10 Khan AJ, Poppe MM, Goyal S, et al: Hypofractionated postmastectomy radiation therapy is safe and effective: first results from a prospective phase II trial. J Clin Oncol 2017;35:2037-2043.

11 Guenzi M, Blandino G, Vidili MG, et al: Hypofractionated irradiation of infra-supraclavicular lymph nodes after axillary dissection in patients with breast cancer post-conservative surgery: impact on late toxicity. Radiat Oncol 2015;10:177.
2 Badiyan SN, Shah C, Arthur D, Khan AJ, Freedman G, Poppe MM, Vicini FA: Hypofractionated regional nodal irradiation for breast cancer: examining the data and potential for future studies. Radiother Oncol 2014; 110:39-44.

13 Bartelink H, Maingon P, Poortmans P, et al.; European Organisation for Research and Treatment of Cancer Radiation Oncology and Breast Cancer Groups: Whole-breast irradiation with or without a boost for patients treated with breast-conserving surgery for early breast cancer: 20 -year follow-up of a randomised phase 3 trial. Lancet Oncol 2015;16:47-56.

14 Romestaing P, Lehingue Y, Carrie C, et al: Role of a $10-\mathrm{Gy}$ boost in the conservative treatment of early breast cancer: results of a randomized clinical trial in Lyon, France. J Clin Oncol 1997;15:963-968.

15 Vrieling C, Collette L, Fourquet A, et al: The influence of patient, tumor and treatment factors on the cosmetic results after breast-conserving therapy in the EORTC 'boost vs. no boost' trial. EORTC Radiotherapy and Breast Cancer Cooperative Groups. Radiother Oncol 2000;55:219-232.

16 Kindts I, Laenen A, Depuydt T, Weltens C: Tumour bed boost radiotherapy for women after breast-conserving surgery. Cochrane Database Syst Rev 2017;11: CD011987. 
17 Bartelink H, Horiot JC, Poortmans P, et al.; European Organisation for Research and Treatment of Cancer Radiation Oncology and Breast Cancer Groups: Recurrence rates after treatment of breast cancer with standard radiotherapy with or without additional radiation. N Engl J Med 2001;345:1378-1387.

18 Vrieling C, van Werkhoven E, Maingon P, et al.; European Organisation for Research and Treatment of Cancer Radiation Oncology and Breast Cancer Groups: Prognostic factors for local control in breast cancer after long-term follow-up in the EORTC Boost vs No Boost Trial: a randomized clinical trial. JAMA Oncol 2017;3:42-48.

19 Sedlmayer F, Reitsamer R, Wenz F, et al: Intraoperative radiotherapy (IORT) as boost in breast cancer. Radiat Oncol 2017;12:23.

20 Sedlmayer F, Sautter-Bihl ML, Budach W, et al.; Breast Cancer Expert Panel of the German Society of Radiation Oncology (DEGRO): Is the simultaneously integrated boost (SIB) technique for early breast cancer ready to be adopted for routine adjuvant radiotherapy? Statemen of the German and the Austrian Societies of Radiooncology (DEGRO/OGRO). Strahlenther Onkol 2013; 189:193-196.

21 Scorsetti M, Alongi F, Fogliata A, et al: Phase I-II study of hypofractionated simultaneous integrated boost using volumetric modulated arc therapy for adjuvant radiation therapy in breast cancer patients: a report of feasibility and early toxicity results in the first 50 treatments. Radiat Oncol 2012;7:145.

22 Chen GP, Liu F, White J, Vicini FA, Freedman GM, Arthur DW, Li XA: A planning comparison of 7 irradiation options allowed in RTOG 1005 for early-stage breast cancer. Med Dosim 2015;40:21-25.

23 Dellas K, Vonthein R, Zimmer J, et al: Hypofractionation with simultaneous integrated boost for early breast cancer: results of the German multicenter phase II trial (ARO-2010-01). Strahlenther Onkol 2014;190:646-653.

24 Wenz F, Sedlmayer F, Herskind C, et al: Accelerated partial breast irradiation in clinical practice. Breast Care 2015;10:247-252.

25 Leonardi MC, Maisonneuve P, Mastropasqua MG, et al: Accelerated partial breast irradiation with intraoperative electrons: using GEC-ESTRO recommendations as guidance for patient selection. Radiother Oncol 2013; 106:21-27.

26 Krug D, Baumann R, Budach W, et al.; Breast cancer expert panel of the German Society of Radiation Oncology (DEGRO): Current controversies in radiotherapy for breast cancer. Radiat Oncol 2017;12:25.

-27 Polgar C, Ott OJ, Hildebrandt G, et al.; Groupe Européen de Curiethérapie of European Society for Radiotherapy and Oncology (GEC-ESTRO): Late side-effects and cosmetic results of accelerated partial breast irradiation with interstitial brachytherapy versus wholebreast irradiation after breast-conserving surgery for low-risk invasive and in-situ carcinoma of the female breast: 5-year results of a randomised, controlled, phase 3 trial. Lancet Oncol 2017;18:259-268.

28 Coles CE, Griffin CL, Kirby AM, et al: Partial-breast radiotherapy after breast conservation surgery for patients with early breast cancer (UK IMPORT LOW trial): 5-year results from a multicentre, randomised, controlled, phase 3, non-inferiority trial. Lancet 2017; 390:1048-1060.

29 Boutrus R, Taghian AG; Association of Radiotherapy and Oncology of the Mediterranean arEa (AROME): Post mastectomy radiation for large node negative breast cancer: time for a second look. Crit Rev Oncol Hematol 2012;84(suppl 1):e75-78.
30 Corradini S, Bauerfeind I, Belka C, et al: Trends in use and outcome of postoperative radiotherapy following mastectomy: a population-based study. Radiother Oncol 2017;122:2-10.

31 Hoffman KE, Mittendorf EA, Buchholz TA: Optimising radiation treatment decisions for patients who receive neoadjuvant chemotherapy and mastectomy. Lancet Oncol 2012;13:e270-276.

32 Wright JL, Takita C, Reis IM, et al: Predictors of locoregional outcome in patients receiving neoadjuvant therapy and postmastectomy radiation. Cancer 2013; 119:16-25

33 Rusthoven CG, Rabinovitch RA, Jones BL, et al: The impact of postmastectomy and regional nodal radiation after neoadjuvant chemotherapy for clinically lymph node-positive breast cancer: a National Cancer Database (NCDB) analysis. Ann Oncol 2016;27:818827.

34 Poortmans PMP, Arenas M, Livi L: Over-irradiation. Breast 2017;31:295-302.

35 Berbers J, van Baardwijk A, Houben R, et al: 'Reconstruction: before or after postmastectomy radiotherapy?' A systematic review of the literature. Eur J Cancer 2014;50:2752-2762

36 Pazos M, Corradini S, Dian D, et al: Neoadjuvant radiotherapy followed by mastectomy and immediate breast reconstruction: an alternative treatment option for locally advanced breast cancer. Strahlenther Onkol 2017; 193:324-331.

37 Giacalone PL, Rathat G, Daures JP, Benos P, Azria D, Rouleau C: New concept for immediate breast reconstruction for invasive cancers: feasibility, oncological safety and esthetic outcome of post-neoadjuvant therapy immediate breast reconstruction versus delayed breast reconstruction: a prospective pilot study. Breast Cancer Res Treat 2010;122:439-451.

38 Zinzindohoue C, Bertrand P, Michel A, et al: A prospective study on skin-sparing mastectomy for immediate breast reconstruction with latissimus dorsi flap after neoadjuvant chemotherapy and radiotherapy in invasive breast carcinoma. Ann Surg Oncol 2016;23: 2350-2356.

39 Krag DN, Anderson SJ, Julian TB, et al: Sentinellymph-node resection compared with conventional axillary-lymph-node dissection in clinically node-negative patients with breast cancer: overall survival findings from the NSABP B-32 randomised phase 3 trial. Lancet Oncol 2010;11:927-933.

40 Donker M, van Tienhoven G, Straver ME, et al: Radiotherapy or surgery of the axilla after a positive sentinel node in breast cancer (EORTC 10981-22023 AMAROS): a randomised, multicentre, open-label, phase 3 noninferiority trial. Lancet Oncol 2014;15:1303-1310.

41 Giuliano AE, McCall L, Beitsch P, et al: Locoregional recurrence after sentinel lymph node dissection with or without axillary dissection in patients with sentinel lymph node metastases: the American College of Surgeons Oncology Group Z0011 randomized trial. Ann Surg 2010;252:426-432; discussion 432-423.

42 Jagsi R, Chadha M, Moni J, et al: Radiation field design in the ACOSOG Z0011 (Alliance) Trial. J Clin Oncol 2014;32:3600-3606.

43 Whelan TJ, Olivotto IA, Levine MN: Regional nodal ir radiation in early-stage breast cancer. N Engl J Med 2015;373:1878-1879.

44 Poortmans PM, Collette S, Kirkove C, et al.; EORTC Radiation Oncology and Breast Cancer Groups: Internal mammary and medial supraclavicular irradiation in breast cancer. N Engl J Med 2015;373:317-327.

45 Hennequin C, Bossard N, Servagi-Vernat S, et al: Tenyear survival results of a randomized trial of irradiation of internal mammary nodes after mastectomy. Int J Radiat Oncol Biol Phys 2013;86:860-866.
46 Budach W, Bolke E, Kammers K, Gerber PA, NestleKramling C, Matuschek C: Adjuvant radiation therapy of regional lymph nodes in breast cancer - a metaanalysis of randomized trials - an update. Radiat Oncol 2015; 10:258

47 Thorsen LB, Offersen BV, Dano H, et al: DBCG-IMN a population-based cohort study on the effect of internal mammary node irradiation in early node-positive breast cancer. J Clin Oncol 2016;34:314-320.

48 Darby SC, McGale P, Taylor CW, Peto R: Long-term mortality from heart disease and lung cancer after radiotherapy for early breast cancer: prospective cohort study of about 300000 women in US SEER cancer registries. Lancet Oncol 2005;6:557-565.

49 Viren T, Heikkila J, Myllyoja K, Koskela K, Lahtinen T, Seppala J: Tangential volumetric modulated arc therapy technique for left-sided breast cancer radiotherapy. Radiat Oncol 2015;10:79.

50 Pignol J-P, Truong P, Rakovitch E, Sattler MG, Whelan TJ, Olivotto IA: Ten years results of the Canadian breast intensity modulated radiation therapy (IMRT) randomized controlled trial. Radiother Oncol; 121:414-419.

51 Darby SC, Ewertz M, McGale P, et al: Risk of ischemic heart disease in women after radiotherapy for breast cancer. N Engl J Med 2013;368:987-998.

52 Corradini S, Ballhausen H, Weingandt H, et al: Leftsided breast cancer and risks of secondary lung cancer and ischemic heart disease: effects of modern radiotherapy techniques. Strahlenther Onkol 2018;194: 196-205.

53 Korreman SS, Pedersen AN, Nottrup TJ, Specht L, Nystrom H: Breathing adapted radiotherapy for breast cancer: comparison of free breathing gating with the breath-hold technique. Radiother Oncol 2005;76:311318.

54 Hjelstuen MHB, Mjaaland I, Vikstrom J, Dybvik KI: Radiation during deep inspiration allows loco-regional treatment of left breast and axillary-, supraclavicularand internal mammary lymph nodes without compromising target coverage or dose restrictions to organs at risk. Acta Oncol 2012;51:333-344.

55 Schonecker S, Heinz C, Sohn M, et al: Reduction of cardiac and coronary artery doses in irradiation of leftsided breast cancer during inspiration breath hold: a planning study. Strahlenther Onkol 2016;192:750-758.

56 Hughes KS, Schnaper LA, Bellon JR, et al: Lumpectomy plus tamoxifen with or without irradiation in women age 70 years or older with early breast cancer: longterm follow-up of CALGB 9343. J Clin Oncol 2013;31: 2382-2387.

57 Kunkler IH, Williams LJ, Jack WJ, Cameron DA, Dixon JM; PRIME II investigators: Breast-conserving surgery with or without irradiation in women aged 65 years or older with early breast cancer (PRIME II): a randomised controlled trial. Lancet Oncol 2015;16: 266-273.

58 Fiorentino A, Mazzola R, Ricchetti F, Fersino S, Levra NG, Alongi F: Personalized - not omitted - radiation oncology for breast cancer. J Clin Oncol 2015;33:43134314.

59 Eaton BR, Jiang R, Torres MA, Kahn ST, Godette K, Lash TL, Ward KC: Benefit of adjuvant radiotherapy after breast-conserving therapy among elderly women with T1-T2N0 estrogen receptor-negative breast cancer. Cancer 2016;122:3059-3068.

60 Kunkler I: Radiotherapy issues in elderly breast cancer patients. Breast Care 2012;7:453-459. 